\title{
Is renal abscess still a problem?
}

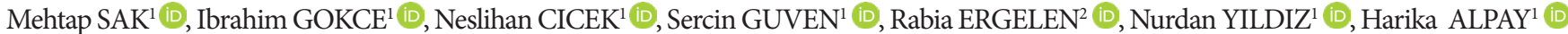

\begin{abstract}
${ }^{1}$ Division of Pediatric Nephrology, Department of Pediatrics, School of Medicine, Marmara University, Istanbul, Turkey.
${ }^{2}$ Department of Radiology, School of Medicine, Marmara University, Istanbul, Turkey.

$\overline{\text { Corresponding Author: Mehtap SAK }}$

E-mail: mehtap_ky@hotmail.com

Submitted: 03.09.2020 Accepted: 30.10.2020

ABSTRACT

Renal abscess, the accumulation of infected fluid in the kidney, is a rare condition seen in children as well as adults. It leads to long term hospital admission and antibiotic use. Early diagnosis is an important factor in the outcome of renal abscess because the management may differ. Urinalysis test results and radiologic imaging findings of the patients who are admitted to hospital with complaints of fever, vomiting, abdominal pain or flank pain are important for the early diagnosis. Undertreated cases have high risk for renal scar formation. In this paper, we aim to present three pediatric patients, who showed the complication of renal abscess and were treated with long term antibiotic use without a need for surgical drainage.

Keywords: Urinary tract infection, Renal abscess, Children
\end{abstract}

\section{INTRODUCTION}

Vesicoureteral reflux (VUR) and recurrent urinary tract infection (UTI) are still among the most common etiologies of endstage renal disease in Turkey. Renal abscess, the accumulation of infected fluid in the kidneys is a rare condition and usually seen in immune-compromised children and children with UTI caused by mostly resistant microorganisms that are not treated properly and promptly. It leads to long term hospital admission and antibiotic use [1]. Lober nefronia, also known as acute focal bacterial nephritis, is the localized infection of the kidney without abscess formation and must be considered in differential diagnosis [2]. Here, we report three cases with renal abscess who were hospitalized and treated with long term antibiotherapy at our hospital in 2018.

\section{CASE REPORTS}

\section{Case 1}

A four-year-old girl was admitted to hospital with fever, abdominal pain and vomiting and did not respond oral antibiotic treatment for 3 days. During physical examination, suprapubic tenderness and fever were present. She had a history of hospitalization because of UTI when she was 3.5 months old. Laboratory findings revealed leukocytosis, mild elevation in renal function tests and pyuria. In urine culture taken by transurethral catheterization $10.000 \mathrm{cfu} / \mathrm{ml}$ Escherichia Coli was present. Renal ultrasonography (US) revealed normal sized kidneys with increased parenchymal echogenicity in the upper pole of the left kidney and perinephritic liquid collection. Abdominal magnetic resonance imaging (MRI) showed a renal abscess measuring 30x27 mm in the left kidney (Figure-1a). Grade 3 VUR to the left kidney and grade 1 VUR to the right kidney were detected in voiding cystourethrography (VCUG) performed on the 26th day of antibiotherapy. Technetium 99m-dimercaptosuccinic acid (99mTc DMSA) renal scan performed on the 16th day of the treatment revealed a well circumscribed lesion in the left kidney, and separated functions were normal. Abscess could not be drained. She was successfully treated with intravenous antibiotic, ceftriaxone treatment initiated first and then teicoplanin was added when urine culture result was available. Treatment was completed in 35 days and abscess formation totally disappeared. Control DMSA scan one year after acute episode showed the disappearance of cortical lesion and revealed only contour deformity in the left kidney, separated functions were still normal. Informed consent form was obtained from her parents. 

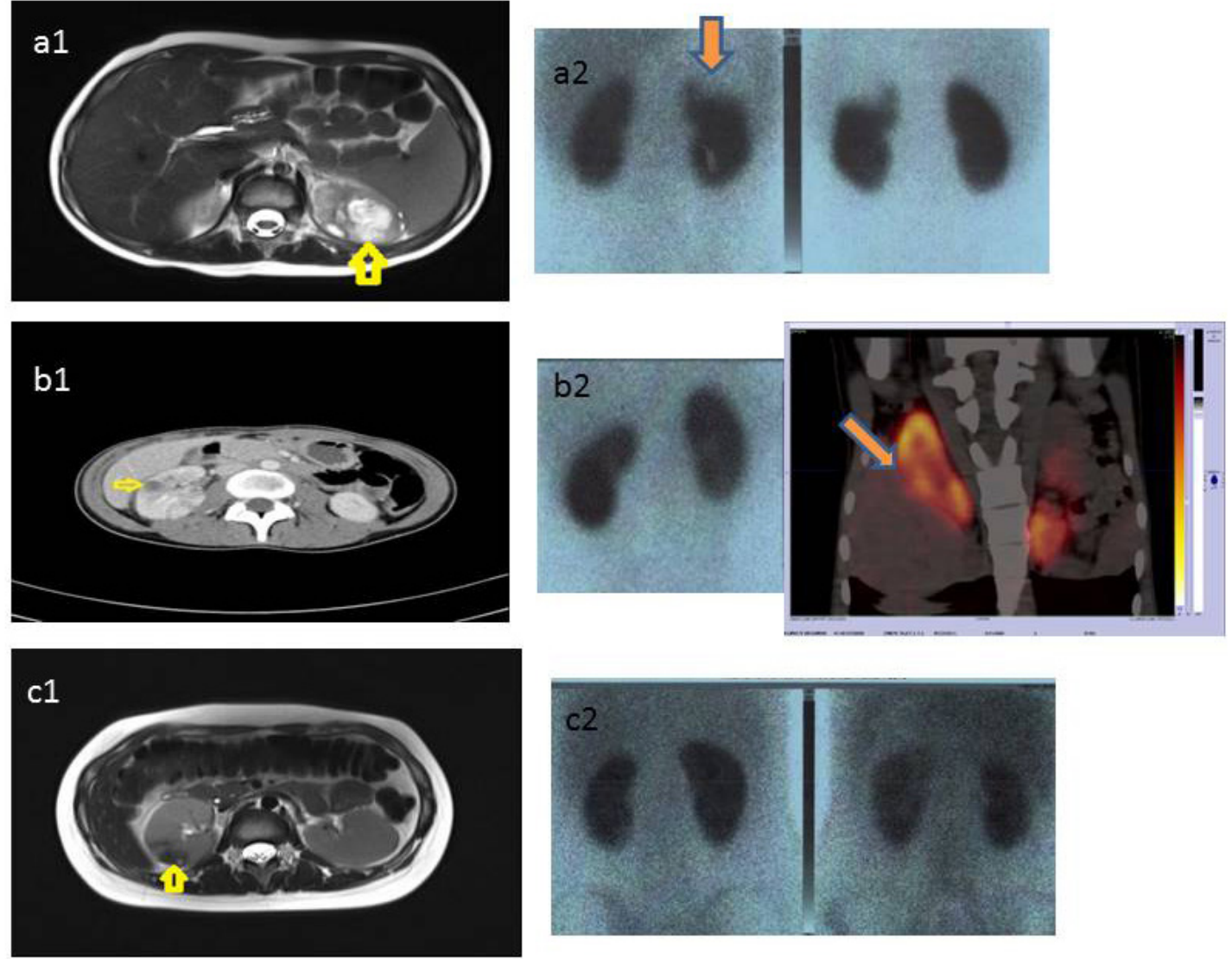

Figure 1. Renal abscess formation of the three cases (arrows). a, c. Abdominal magnetic resonance and DMSA scans of case 1 and 3 respectively b. Computed tomography, DMSA and coronal SPECT CT scans of case 2.

\section{Case 2}

A previously healthy 17 years old girl admitted to hospital with the complaints of nausea, vomiting, fever and malaise for five days. On physical examination, costovertebral angle tenderness was present. Laboratory findings revealed leukocytosis, anemia, mild elevation in renal function tests and pyuria. In urine culture obtained by clean catch method $>100.000 \mathrm{cfu} / \mathrm{ml}$ Escherichia Coli was present. Renal US revealed normal sized kidneys with $15 \mathrm{~mm}$ diameter non perfused area in the middle of the right kidney. Computed tomography showed renal abscess formation measuring about $13 \mathrm{~mm}$ in the right kidney (Figure-1b). She was successfully treated with intravenous ceftriaxone for 14 days and followed by oral cefixime for 7 days. 99mTc DMSA scan was performed on the 7th day of antibiotherapy and it revealed well circumscribed lesion in the right kidney, separated functions were normal. Abscess formation totally disappeared with medical treatment. Control DMSA scan one year later revealed contour deformity in the right kidney but not scarring as we observed in the first patient, separated functions were also normal. Informed consent form was obtained from her parents.

\section{Case 3}

A previously healthy 16 years old girl admitted to hospital with the complaints of nausea, vomiting, fever, malaise and right side flank pain for seven days. On physical examination costovertebral angle tenderness was present. Laboratory findings revealed elevated C-reactive protein (CRP) level. Her creatinine level increased up to $5.6 \mathrm{mg} / \mathrm{dl}$ in the follow up and returned to the normal level without any need to renal replacement therapy. There was no pyuria in urinalysis and urine culture obtained by clean catch method was sterile. Renal US revealed normal sized kidneys with renal abscess formation in the right kidney. Abdominal MRI showed renal abscess measuring 40x35 mm (Figure-1c). VCUG performed on the 11th day of antibioteraphy was normal. 99mTc DMSA renal scan performed on the 17th day of the treatment and did not reveal cortical scarring but background activity was increased. She was first treated with ceftriaxone, and then treatment upgraded to piperacillin and vancomycin according to urine culture result and finally switched to meropenem when she developed acute kidney injury. Abscess formation totally disappeared with medical treatment administered for 28 days. Control DMSA scan one year later revealed hypoactive area in the upper pole of the right 
kidney, separated functions were normal. Informed consent form was obtained from her parents.

\section{DISCUSSION}

Urinary tract infection is one of the most common bacterial infections in children especially in infancy and mostly it is uncomplicated and can be treated by oral or iv antibiotics. It ranges from lower urinary tract infections to acute piyelonephritis (APN), acute lober nephronia and renal abscess. The association between UTI and congenital abnormalities, like VUR, may put children at high risk for APN and subsequent renal scarring [3].

Acute lober nephronia, also known as acute focal bacterial nephritis (AFBN) was first radiologically described in 1979 [4]. It is an acute, localized non-liquefactive infection of the kidney caused by bacterial infection generally affecting one or more renal lobules. It is considered as at the midpoint in the spectrum of upper UTI between APN and intrarenal abscess [5]. The diagnosis of AFBN is dependent on radiologic imaging. Acute focal bacterial nephritis is characterized by hypoperfused wedged-shaped or round and space-occupying lesions in the kidney, exhibiting no capsule [6-8]. Acute focal bacterial nephritis is associated with higher incidence of renal scarring in comparison to APN. It must be treated by long course intravenous antibiotics at least 2 to 3 weeks [9]. Bitsori, et al., evaluated 25 pediatric patients (27 episodes), 21 with AFBN, one with abscess and three with pyonephrosis and suggested at least 1 week of intravenous treatment and a total antibiotic course of 3 weeks in patients with AFBN [10].

Renal abscess is the most complicated form of UTI. In renal abscess, the infected material is localized in the parenchyma. It is often caused by ascending infections of the lower urinary tract by gram negative organisms or hematogenic spread. Clinical symptoms are similar to APN and AFBN. Laboratory investigation reveals leukocytosis and elevated CRP levels [11]. Our first two patients both had leukocytosis and elevated CRP levels, third patient only had elevated CRP level. Urinalysis generally shows pyuria or may be normal if it is caused by hematogenic spread or if the patient received antibiotics before performing urinalysis and urine culture. There may or may not be growth in the urine culture test. Bitsori, et al., demonstrated abnormal urinalysis in 16 (59\%) and Eschericia coli bacteriuria as a leading pathogen in $12(44 \%)$ episodes [10]. Similarly, in our two patients Escherichia Coli was the predisposing cause of renal abscess formation. The urinalysis of our third patient was also normal and urine culture was sterile. Our patient did not have a history of receiving antibiotic treatment before performing urinalysis and her blood culture was also sterile excluding hematogenic spread.

Renal and perirenal abscess management includes medical treatment, percutaneous drainage, surgical drainage (open surgery), and nephrectomy. Dalla Palma, et al. and Lee, et al., confirmed that renal abscesses of $50 \mathrm{~mm}$ diameter or less can completely regress after long term medical treatment. Although, antibiotic therapy is the mainstay of the treatment, percutaneous or surgical drainage of abscesses larger than $50 \mathrm{~mm}$ in diameter is suggested. According to some authors, drainage treatment of the abscesses $30-50 \mathrm{~mm}$ in diameter should be planned, especially if there is no response to the antibiotic treatment [12, 13]. Similarly, all of our patients were successfully treated by long term antibiotic use. None of them needed invasive surgical treatments.

Acute interstitial nephritis (AIN) is a common cause of acute kidney injury (AKI) associated with drugs, infections or unknown causes. AIN associated with infections constitute a proportion of \%5-10 of all AIN cases and its frequency decreased after widespread of antibiotic use. Bitsori, et al., revealed a reversible increase of creatinine in 15 (55\%) episodes [10]. The gold standard of diagnosis of AIN is kidney biopsy. Treatment consists supportive therapy and treating underlying infections, it has been reported that steroid treatment is beneficial for total recovery [14]. Our third patient's creatinine level also increased up to $5 \mathrm{mg} / \mathrm{dl}$ in the follow up and recovered by supportive treatment. We suspected infection associated AIN for this patient. Since the renal functions of the patient quickly regressed, we did not perform a renal biopsy to confirm it.

In conclusion, early diagnosis is an important factor in the outcome of renal abscess. Urinalysis and radiologic imaging of the patients who admitted to hospital with complaints of fever, vomiting, abdominal pain or flank pain is important for the early diagnosis. It should be kept in mind that we cannot exclude renal abscess even if the urinalysis is normal. Undertreated cases have the risk for development of renal scarring.

\section{Compliance with the Ethical Standards}

Written informed consents were obtained from the parents for publication of these case reports and accompanying images.

Financial Support: The authors have no relevant financial information to disclose.

Conflict of Interest: The authors have no potential conflicts to disclose.

Author Contributions: M.S. : Coordinated and supervised data collection, drafted the initial manuscript, reviewed and revised the manuscript, I.G.: Conceptualized and designed the cases, coordinated and supervised data collection, N.C., S.G. and N.Y. : Had active roles in the diagnosis and treatment of the patients, R.E.: Evaluated radiological images of the patients, H. A. : Critically reviewed and revised the manuscript. All authors read and approved the final version of the article.

\section{REFERENCES}

[1] Chen CY, Kuo HT, Chang YJ, Wu KH, Yang WC, Wu HP. Clinical assessment of children with renal abscesses presenting to the pediatric emergency department. BMC Pediatr 2016; 16: 189. doi: 10.1186/s12887.016.0732-5.

[2] Bibalo C, Apicella A, Guastalla V, et al. Acute lobar nephritis in children: Not so easy to recognize and manage. World J Clin Pediatr 2016; 5: 136-42. doi: 10.5409/wjcp.v5.i1.136. 
[3] Sheu JN, Wu KH, Chen SM, Tsai JD, Chao YH, Lue KH. Acute 99mTc DMSA scan predicts dilating vesicoureteral reflux in young children with a first febrile urinary tract infection: a population-based cohort study. Clin Nucl Med 2013; 38: 16368. doi: 10.1097/RLU.0b013e318279f112.

[4] Rosenfield AT, Glickman MG, Taylor KJ, Crade M, Hodson J. Acute focal bacterial nephritis (acute lobar nephronia). Radiology 1979; 132: 553-61. doi: 10.1148/132.3.553.

[5] Sheu JN. Acute Lobar Nephronia in Children. Pediatrics and Neonatology 2015; 56: 141 - 42. doi: 10.1016/j. pedneo.2015.03.001.

[6] Nosher JL, Tamminen JL, Amorosa JK, Kallich M. Acute focal bacterial nephritis. Am J Kidney Dis 1988; 11: 36-42. doi: 10.1016/s0272-6386(88)80172-0.

[7] Lee JK, McClennan BL, Melson GL, Stanley RJ. Acute focal bacterial nephritis: emphasis on gray scale sonography and computed tomography. AJR Am J Roentgenol 1980; 135: 8792. doi: 10.2214/ajr.135.1.87.

[8] Choyke PL, Kressel HY, Pollack HM, Arger PM, Axel L, Mamourian AC. Focal renal masses: magnetic resonance imaging. Radiology 1984; 152: 471-7. doi: 10.1148/radiology. 152.2.6739818.
[9] Sieger N, Kyriazis I, Schaudinn A, et al. Acute focal bacterial nephritis is associated with invasive diagnostic procedures a cohort of 138 cases extracted through a systematic review. BMC Infect Dis 2017; 17: 240. doi: 10.1186/s12879.017.23366.

[10] Bitsori M, Raissaki M, Maraki S, Galanakis E. Acute focal bacterial nephritis, pyonephrosis and renal abscess in children. Pediatr Nephrol 2015; 30: 1987-93. doi: 10.1007/ s00467.015.3141-3.

[11] Rubilotta E, Balzarro M, Lacola V, Sarti A, Porcaro A.B, Artibani W. Current clinical management of renal and perinephric abscesses. Urologia 2014; 81: 144-7. doi: 10.5301/ urologia.5000044.

[12] Dalla Palma L, Pozzi Mucelli F, Ene V. Medical treatment of renal and perirenal abscesses: CT evaluation. Clin Radiol 1999; 54: 792-7. doi:10.1016/s0009-9260(99)90680-3.

[13] Lee SH, Jung HJ, Mah SY, Chung BH. Renal abscesses measuring $5 \mathrm{~cm}$ or less: outcome of medical treatment without therapeutic drainage. Yonsei Med J 2010; 4: 569-73. doi: 10.3349/ymj.2010.51.4.569.

[14] Raghavan R, Eknoyan G. Acute interstitial nephritis - a reappraisal and update. Clin Nephrol 2014; 82: 149-62. doi: $10.5414 / \mathrm{cn} 108386$. 